\title{
Evaluation ON OVERLAPPING BARRIERS DESIGN USING SOUNDPLAN
}

\author{
Sheying Sun ${ }^{\star 1}$ and Samuel B. Lacrampe ${ }^{\dagger 1}$ \\ ${ }^{1}$ Patching Associates Acoustical Engineering Ltd., Calgary, Alberta, Canada.
}

\section{Introduction}

Traffic noise comes along with highway and city road developments. As these roadways are being built closer and closer to residential areas, residents will be exposed to higher or even intolerable noise levels. To overcome traffic noise problems, solutions such as noise barriers, low noise pavements, low noise vehicles, traffic control measures, and proper land uses have been proposed.

Due to being considered to have great benefits of easy installation, better noise reduction performance, and ability to soothe annoyed residents, noise barriers have become the most prevalent noise control measures adopted by most agencies. However any defects in noise barriers may allow unnecessary noise propagation and thus degrade their performance. Consequently, noise barriers should be constructed and maintained with care to uphold their designed noise reduction capability.

Ideally, noise barriers would have no breaks at all. However, few barriers can be designed without the need for a break in the wall. Breaks in noise barriers are needed for many reasons, such as maintenance, safety access, drainage, and barrier transitions between different roadway sections. When these breaks are necessary, overlap gaps are often used to lessen the effect of the discontinuity, but inappropriate design may cause severe degradation of acoustical effectiveness, and therefore, acquiring the appropriate parameters of the overlapping barrier design will serve as a reference for decision makers to properly allocate the gap location and select the sound design.

This analysis focuses on the evaluation of overlapping barrier design and receiver regions in the vicinity of an overlap gap. Noise attenuation performance of overlapping barrier from road traffic noise was investigated using the FHWA TNM version 2.5 module in SoundPlan v7.4 computer program, which not only corrects known deficiencies in the TNM algorithms, but also considers the reflection effects of the barrier [1,2]. The contributions from various parameters such as materials, barrier height, gap, and overlapping sizes were investigated.

\section{Gap geometry and propagation paths}

The possible paths through which noise may propagate to a receiver at an overlap gap situation are: direct propagation, transmission, simple diffraction, double diffraction, multiple reflections, and multiple reflected diffractions.

In order to study the effect of overlap gaps on the surrounding area, a simplified noise model was created as per the typical overlap gap configuration, which is represented in Figure 1. The traffic noise source is taken to be the center of road. Receivers being investigated can be located within the gap or more typically on the opposite side of the barrier from the source. Due to the complications of sound propagation mechanism, a number of simplifications, assumptions, and/or limitations were used in the development of the noise model. All barriers were considered to be parallel to the line of the road with a typical height of $1.8 \mathrm{~m}$, which is similar to a regular private fence. Ten reflection orders were considered to be sufficient reflection inside the gap area. Ground is assumed to be typical lawn area. The road was modelled as 1000 vehicles per hour with $110 \mathrm{~km} / \mathrm{hr}$ speed. The area was assumed as flat. The barrier materials were considered as five types of absorption, which includes totally reflective, smooth façade, structural façade, absorptive material and highly absorptive material (each with corresponding reflection loss $0,1,2,4$, $8 \mathrm{~dB}$, named as RL 0, RL 1, RL 2, RL 4, RL 8).

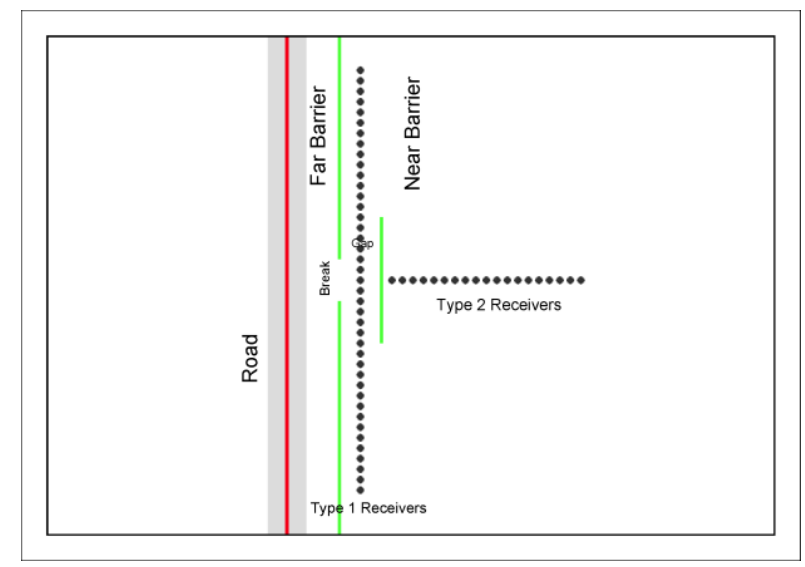

Figure 1: Typical overlap gap configuration

There are certain design characteristics that must be considered to ensure a barrier's optimum performance. The barrier must be sufficiently long so that the receiver is protected from edge diffraction. Edge diffraction is the propagation of sound waves around the ends of a noise barrier. If a barrier is extended a reasonable distance past any potential receiver, edge diffraction is considered to have minimal effect on the noise level. In addition, the noise barrier must be constructed from a solid material of adequate density with no cracks to prevent noise from transmitting directly through the barrier. With that considered, the effectiveness of a noise barrier depends directly on its length and its heights with respect to the source and receiver locations. 


\section{Barrier performance investigation}

\subsection{Single barrier performance}

In order to provide a basis to judge the effectiveness of an overlap gap design, insertion loss of a single barrier is investigated. First, an analysis was done on the single noise barrier to determine the noise levels at a receiver's position without the break, and different absorption types. Figures $2 \mathrm{a}$ and $2 b$ show that sound attenuation of the single barrier related to the barrier absorption for the two types of receivers. The results indicate that barrier absorption benefit is minor in this single barrier scenario, which is usually less than $1 \mathrm{~dB}$ additional insertion loss.

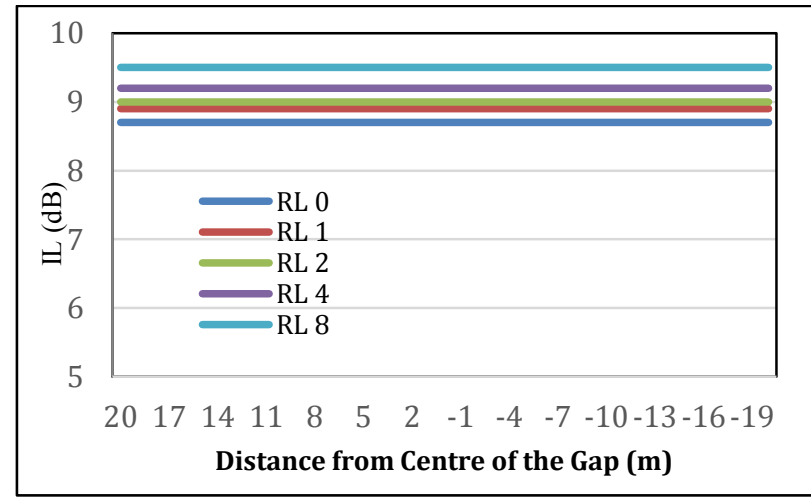

Figure 2a: Sound attenuation of the single barrier related to the barrier absorption for type 1 receivers

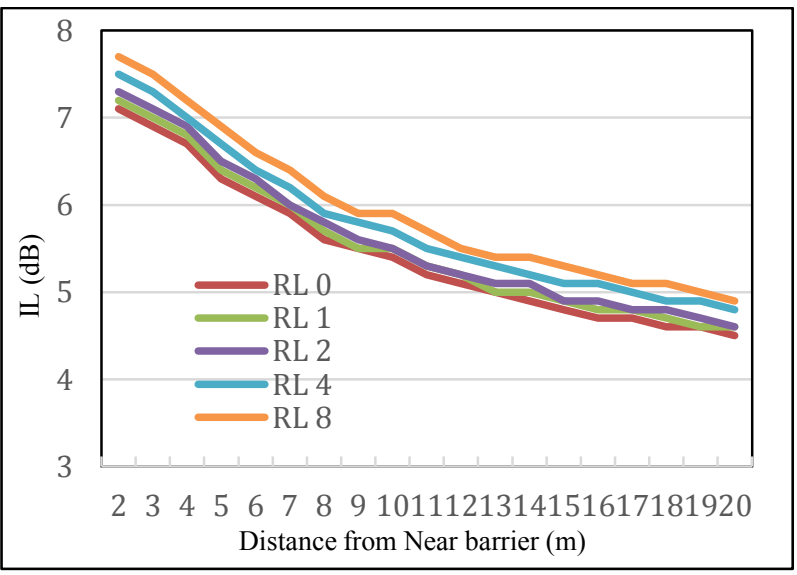

Figure 2b: Sound attenuation of the single barrier related to the barrier absorption for type 2 receivers

\subsection{Overlapping gap barrier}

To reduce the negative effect that this gap has on the noise environment, the ends of each barrier are extended past one another to form an overlap. This blocks line-of-sight propagation for most propagation paths. The amount of overlap is often selected to reduce the levels of noise flanking around the ends of the barriers to the point that the noise diffracting over the top of the barrier becomes the dominant noise at the receiver.
The primary problem with installing an overlap gap is the introduction of reflective sound waves. By overlapping the near and far barriers a sufficient distance, the line-ofsight is broken between the traffic and most receivers; and therefore, the majority of direct rays are eliminated. However, reflected rays exist regardless of the length of the overlap. Absorptive treatment is commonly used on parallel noise barriers to prevent reflections from causing insertion loss degradation.

Research has been conducted to study the effectiveness of using absorptive panels to minimize reflections at overlap gap barriers. Insertion loss is investigated with different absorption type inside the gap surfaces. The results for the type 1 receivers show that a higher absorption inside the gap is efficient to attenuate the noise, and the overlapping length should be at least the same length of the break size. Figure 3 shows the results with this typical gap design.

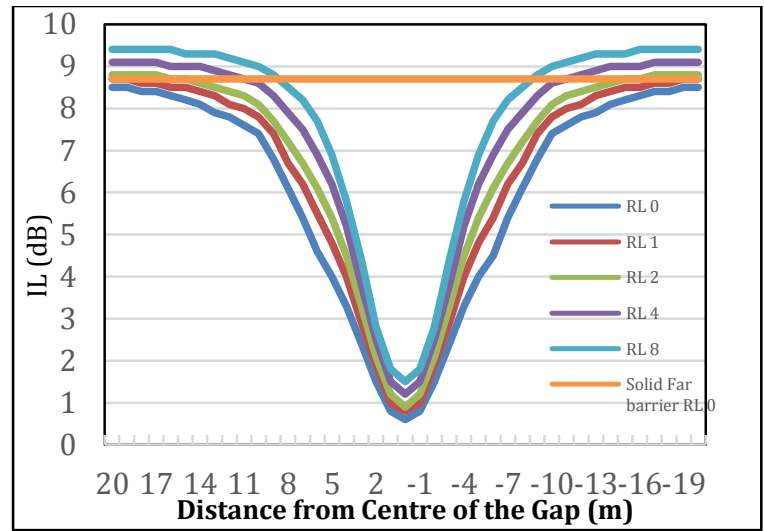

Figure 3: Sound attenuation of the overlapping barrier related to the barrier absorption

The results indicate that the insertion loss with the absorption can be less than $2 \mathrm{dBA}$ at the gap end of the overlapping barrier, compared to a far barrier with no break.

\section{Conclusion}

This study first investigated the solid barrier performance with the absorption and insertion loss degradation with break. The results showed that an absorption benefit for the barrier performance is minor, which is usually less than 1 $\mathrm{dB}$ additional insertion loss.

For this scenario, the overlapping length should be at least the same length of the break size with this typical overlap gap design, which means the total length of the near barrier would be 3 times of the break size. The results indicate that insertion loss with the absorption can be less than $2 \mathrm{dBA}$ at the gap end of the overlapping barrier, compared to a barrier with no break.

\section{References}

[1] U.S. Department of Tranportation, Federal Highway Administration; Traffic Noise Model (FWHA TNM) Technical manual. 1998

[2] Braunstein + Berndt GmbH/SoundPLAN International LLC. SoundPLAN User's Manual, February 2014 